\section{Caracterização da Estratégia Saúde da Família no Estado do Amazonas, Brasil: análise da implantação e impacto}

\author{
Characterization of the family health strategy \\ in Amazonas State, Brazil: an analysis of \\ implementation and impact
}

\author{
1 Escola de Enfermagem \\ de Manaus, Universidade \\ Federal do Amazonas, \\ Manaus, Brasil. \\ 2 Programa de Pós-graduação \\ Multi-institucional em \\ Saúde, Sociedade e Endemias \\ na Amazônia, Universidade \\ Federal do Pará/Universidade \\ Federal do Amazonas/Centro \\ de Pesquisas Leônidas e \\ Maria Deane, Fundação \\ Oswaldo Cruz, Manaus, \\ Brasil. \\ ${ }^{3}$ Centro de Ciências da \\ Saúde, Universidade \\ Federal de Santa Catarina \\ Florianópolis, Brasil. \\ Correspondência \\ H. M. Oliveira \\ Escola de Enfermagem de \\ Manaus, Universidade Federal \\ do Amazonas. \\ Rua Teresina 495, Manaus, \\ AM 69057-070, Brasil. \\ hmilon@ufam.edu.br
}

\begin{abstract}
This article focuses on the implementation of the Family Health Program (FHP) in municipalities (counties) in the State of Amazonas, Brazil, analyzing potential population coverage, evidence of change in the health care model, and impact (2004 and 2008). Implementation was classified as fair versus unsatisfactory. The findings showed an increase in the number of municipalities with the FHP implemented, as well as in the program's coverage (which nevertheless was less than 50\% of the population). Concerning change in the health care model, no municipalities were observed with a high rate of change. As for impact, there was a decrease in low impact and an increase in high impact. The final classification did not show a statistically significant change from one year to the next, and unsatisfactory implementation prevailed in the municipalities of the State of Amazonas. Evaluation of the FHP is not simple, since there are various factors involved in the issue. Further studies could reveal the factors that explain the program's suboptimal implementation in the State of Amazonas.
\end{abstract}

Family Health Program; Program Evaluation; Health Evaluation; Health Services Coverage
Hadelândia Milon de Oliveira 1,2 Maria Jacirema Ferreira Gonçalves 1,2 Rodrigo Otávio Moretti Pires 2,3

\section{Introdução}

Desde sua criação, o Programa Saúde da Família (PSF) é uma estratégia que vem se estendendo no território nacional. Sua área de cobertura assistencial tem sido ampliada, seguindo uma tendência de expansão em áreas menos assistidas, afastadas dos grandes centros urbanos, representadas por pequenos municípios. São inegáveis os avanços nessa expansão, entretanto o crescimento do número de equipes não implica, necessariamente, uma alteração real das tradicionais formas de atenção à saúde ou uma estratégia de promoção de equidade 1,2. Alguns problemas são evidentes, como as condições físicas das unidades 2 .

No Estado do Amazonas, a implantação do PSF, em 2008, contou com 386 equipes de saúde da família, uma cobertura populacional de mais de $40 \%$. Cerca de 1,316 milhão de pessoas foram atendidas pelo programa no estado em 2008 (Ministério da Saúde. http://dtr2004.saude.gov. br/dab/histórico_cobertura_sf_relatorio.php, acessado em 01/Jun/2009).

Não foram encontrados estudos na literatura científica sobre como o PSF tem sido implantado e desenvolvido no Amazonas. O estado, o maior da Federação, com 1.577.820,2km² e 1,79 habitantes $/ \mathrm{km}^{2}$, conta com características geográficas desfavoráveis ao acesso fácil aos serviços de saúde. Apresenta problemas de saneamento básico e desigualdades econômicas, além 
de uma população com características variadas em áreas urbanas e rurais, sendo ribeirinhos ou indígenas (Instituto Brasileiro de Geografia e Estatística.Cidades@.http://www.ibge.gov.br/ cidadesat/topwindow.htm?1, acessado em 01/ Jun/2009). A população está em contato com doenças predominantes de clima tropical úmido, o que, somando-se às condições acima descritas, deixam-na vulnerável a diversos problemas de saúde.

O PSF, criado no ano de 1994, apresenta-se como reformulação do modelo assistencial de saúde no Brasil. Tem como base os princípios que regem o Sistema Único de Saúde (SUS) e como foco, o trabalho com a família e a comunidade 1. Portanto, este trabalho tem como objetivo caracterizar a implantação do PSF nos municípios do Estado do Amazonas, analisando-se os anos de 2004 e 2008, por meio de indicadores representativos de cobertura, indícios de mudança do modelo assistencial e impacto.

As nomenclaturas PSF e Estratégia Saúde da Família são utilizadas pelo Ministério da Saúde para descrever o mesmo programa. Neste trabalho será utilizado o termo PSF.

\section{Métodos}

Estudo epidemiológico, ecológico, quantitativo, baseado em dados secundários obtidos no Sistema de Informação da Atenção Básica - SIAB (Departamento de Informática do SUS. http:// tabnet.datasus.gov.br/cgi/deftohtm.exe?sia/ cnv/paam.def, acessado em 01/Jun/2006; Ministério da Saúde. http://dtr2004.saude.gov. br/dab/histórico_cobertura_sf_relatorio.php, acessado em 01/Jun/2009) e no Sistema de Informações Hospitalares - SIH (Departamento de Informática do SUS. http://tabnet.datasus. gov/deftohtm.exe?sih/cnv/mram.htm, acessado em 05/Jun/2009), de todos os municípios do Amazonas apresentados nos sistemas em 2004 e 2008. Os dados foram coletados nos mês de junho de 2009 .

Os métodos e técnicas utilizados seguem a proposta desenvolvida por Henrique \& Calvo ${ }^{3}$, em trabalho que analisou dados do Estado de Santa Catarina.

Foram utilizados indicadores que compõem as seguintes dimensões: cobertura, mudança no modelo assistencial e impacto.

A análise da dimensão cobertura baseou-se no cálculo do percentual de cobertura populacional potencial. Esse cálculo representa os recursos disponíveis para atender de forma satisfatória a população adscrita, e não para a que efetivamente utilizou o PSF. Para o cálculo da cobertura populacional potencial, utilizou-se a fórmula proposta pelo Ministério da Saúde 1:

Cobertura populacional potencial $=$

Número de equipes x 3.450 $\mathrm{x} 100$

Número de residentes no município

A cobertura potencial foi categorizada em: alta (maior que 70\%), média (entre 50\% e 70\%) e baixa (menor que $50 \%$ ) 7 .

Para a dimensão de mudanças no modelo assistencial, foram utilizados dados sobre as atividades desenvolvidas pela equipe do PSF, também obtidos nos sistemas de informações supramencionados. Essa verificação teve como base o número de visitas domiciliares do médico, número de exames de patologia clínica, número de encaminhamentos ao especialista e o número de atendimentos individuais do enfermeiro.

Para o indicador de indício de mudança foram utilizadas internações por algumas doenças sensíveis à atenção básica, sendo coletados os dados com base na lista de morbidade da Classificação Internacional de Doenças e Problemas Relacionados à Saúde - 10 a Revisão (CID-10) 4. Para o indicador das causas de internações, utilizou-se o somatório das doenças, conforme o estudo de Henrique \& Calvo ${ }^{3}$, quais sejam: asma (J45), insuficiência cardíaca (I50), pneumonia (J12 a J18) e diarréia e gastroenterite de origem infecciosa presumível (A09). Constituiu-se a taxa de internações por algumas doenças sensíveis à atenção primária, para um conjunto de mil habitantes, no período de um ano, dividindo-se por tercis: baixo, médio e alto número de internações.

A Figura 1 traz um demonstrativo da descrição das variáveis, as medidas e os parâmetros para a classificação dos municípios do Estado do Amazonas em 2004 e 2008, respectivamente. Para a atribuição de escores, foi empregada a mesma metodologia de Henrique \& Calvo ${ }^{3}$ : para cada município, foi atribuído escore 1 (um) no aspecto analisado cujo valor estivesse dentro do intervalo esperado e escore 0 (zero) para valores acima ou abaixo deste intervalo, uma vez que valores fora do esperado (acima ou abaixo) podem representar desequilíbrio do modelo de atenção, ou uma rede de atenção mal estruturada, com excesso ou falta de oferta de determinados serviços.

Os dados foram analisados no pacote estatístico Stata 9.0 (Stata Corp., College Station, Estados Unidos). Os indicadores foram agrupados de modo a permitir a classificação de implantação intermediária, satisfatória. O teste do qui-quadrado $\left(\chi^{2}\right)$ foi utilizado para identificar associação entre as classificações obtidas nos anos de 2004 e 2008, com a respectiva análise dos resíduos padronizados 5 . O qui-quadrado geral indica 
Diagrama dos componentes da análise: indicadores, medidas, parâmetros, escores e classificação final.

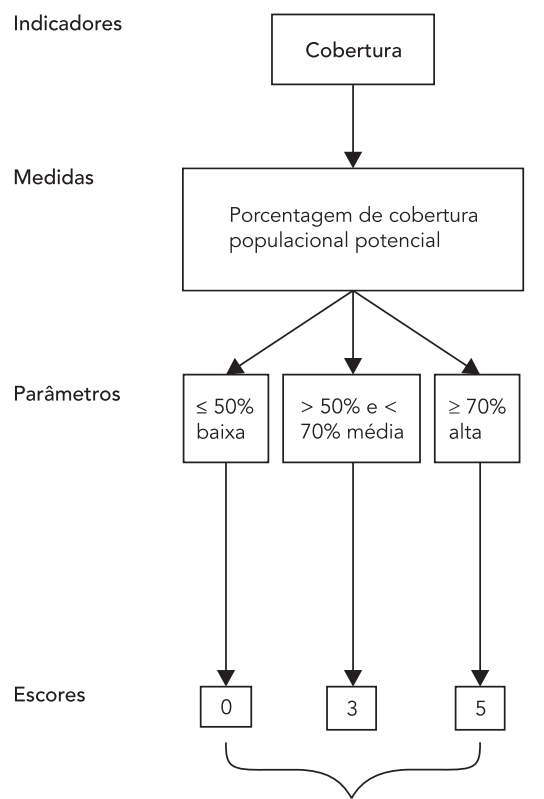

Classificação final

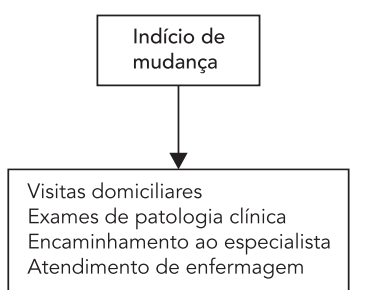

Atendimento de enfermagem

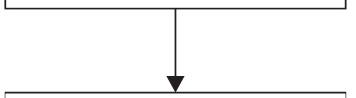

1 visita domiciliar por 12-24 consultas médicas

1 exame de patologia clínica por 3, 1-5, 4 consultas médicas ** 1 encaminhamento ao especialista por $5,0-10,0$ consultas médicas 1 atendimento de enfermagem por 2, 0-3, 9 consultas médicas **
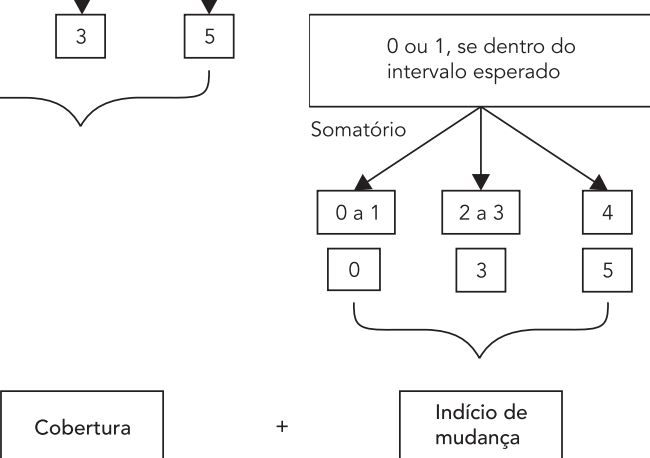

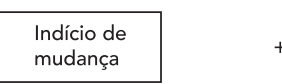

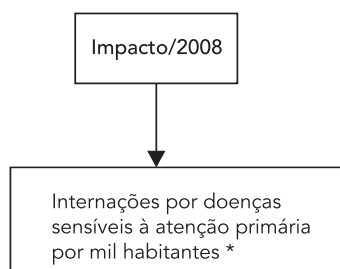
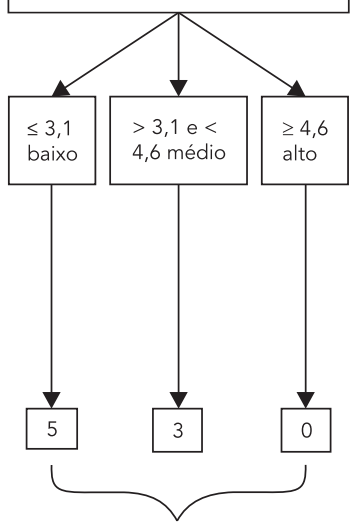

Impacto/2004

Impacto/2008

< 7 (Insatisfatório) 7-10 (Intermediário) $>10$ (Satisfatório)

* Os parâmetros de internação apresentam tercis diferentes em 2004 e 2008;

** Os intervalos são os valores esperados para as variáveis. Valores acima ou abaixo do esperado podem representar desequilíbrio do modelo de atenção. Adaptado de Henrique \& Calvo 3

Fonte: Sistema de Informação da Atenção Básica e Sistema de Informações Hospitalares do Departamento de Informática do SUS.

se a distribuição das categorias é dependente, e os resíduos padronizados revelam os padrões característicos de cada categoria de classificação nos anos de estudo, segundo o excesso ou falta de ocorrências, permitindo concluir a respeito da significância das associações. O nível de significância adotado foi de $5 \%$ para a associação entre as variáveis no teste do $\chi^{2}$; na análise de resíduos, a significância para o excesso de ocorrências corresponde ao resíduo com valor positivo superior a 1,96.

Para o mapeamento da classificação final, foi utilizado o programa ArcView versão 3.3 (Environmental Systems Research Institute Inc., http://www.esri.com/software/arcview/).

\section{Resultados}

Os dados foram organizados pelas dimensões, a fim de proporcionar a classificação do PSF no Amazonas. A Tabela 1 apresenta os resultados dos indicadores e das variáveis por municípios, nos anos de 2004 e 2008.

Na Tabela 2, é possível visualizar a classificação dos indicadores e o teste qui-quadrado com os resíduos padronizados, indicando as categorias onde houve diferença nos os anos de estudo.

No ano de 2004, a cobertura populacional na maioria dos municípios amazonenses $(n=46)$ era baixa, e apenas oito municípios apresentavam 
Distribuição dos indicadores e classificação da implantação do Programa Saúde da Família nos municípios do Amazonas, Brasil, 2004 e 2008.

\begin{tabular}{|c|c|c|c|c|c|c|c|c|c|c|c|c|c|c|}
\hline \multirow[t]{2}{*}{ Municípios } & \multicolumn{2}{|c|}{$\begin{array}{l}\text { Cobertura } \\
\text { populacional } \\
\text { potencial } \\
(\%)\end{array}$} & \multicolumn{2}{|c|}{$\begin{array}{l}\text { Razão da visita } \\
\text { domiciliar do } \\
\text { médico }\end{array}$} & \multicolumn{4}{|c|}{ Dimensão mudança * } & \multicolumn{2}{|c|}{$\begin{array}{c}\text { Razão de } \\
\text { ○ atendimento } \\
\text { de } \\
\text { enfermagem }\end{array}$} & \multicolumn{2}{|c|}{$\begin{array}{c}\text { Dimensão impacto } \\
\text { Número de } \\
\text { internações/ } \\
1.000 \text { habitantes }\end{array}$} & \multicolumn{2}{|c|}{$\begin{array}{l}\text { Classificação da } \\
\text { implantação }\end{array}$} \\
\hline & 2004 & 2008 & 2004 & 2008 & 2004 & 2008 & 2004 & 2008 & 2004 & 2008 & 2004 & 2008 & 2004 & 2008 \\
\hline Amaturá & 0,0 & 0,0 & 0,0 & 0,0 & 0,0 & 0,0 & 0,0 & 0,0 & 0,0 & 0,0 & 8,4 & 24,0 & Insatisfatória & Insatisfatória \\
\hline Anamã & 0,0 & 49,7 & 0,0 & 30,3 & 0,0 & 22,6 & 0,0 & 62,7 & 0,0 & 0,9 & 10,6 & 22,3 & Insatisfatória & Insatisfatória \\
\hline Anori & 29,2 & 79,7 & 31,4 & 2,4 & 10,3 & 1,8 & 816,3 & 34,5 & 24,7 & 0,3 & 5,6 & 3,7 & Insatisfatória & Intermediária \\
\hline Apuí & 0,0 & 59,5 & 108,3 & 3,8 & 6,6 & 0,5 & 383,9 & 165,7 & $1.111,2$ & 0,2 & 7,4 & 6,4 & Insatisfatória & Insatisfatória \\
\hline Atalaia do Norte & 32,9 & 27,7 & 21,9 & 26,9 & 8,1 & 0,8 & 0,0 & 0,0 & 3,3 & 0,8 & 3,1 & 4,7 & Intermediária & Insatisfatória \\
\hline Benjamin & 0,0 & 25,7 & 20,7 & 22,3 & 4,5 & 3,1 & 78,7 & 4,8 & 6,2 & 0,6 & 1,6 & 3,9 & Intermediária & Insatisfatória \\
\hline Constant & & & & & & & & & & & & & & \\
\hline Beruri & 0,0 & 24,6 & 0,0 & 90,1 & 0,0 & 0,6 & 0,0 & 41,3 & 0,0 & 2,0 & 1,8 & 1,6 & Insatisfatória & Insatisfatória \\
\hline Boa Vista do & 31,1 & 54,7 & $1.199,5$ & 4,4 & 261,0 & 1,5 & 0,0 & 921,1 & $1.232,1$ & 2,2 & 3,6 & 4,7 & Insatisfatória & Insatisfatória \\
\hline Ramos & & & & & & & & & & & & & & \\
\hline Boca do Acre & 63,0 & 64,0 & 19,2 & 1,6 & 12,1 & 0,3 & 0,2 & 15,4 & 4,1 & 0,5 & 2,5 & 1,8 & Intermediária & Intermediária \\
\hline Borba & 22,3 & 81,0 & 30,2 & 1,3 & $2.596,8$ & 0,8 & 349,5 & 133,5 & 992,4 & 0,2 & 3,6 & 6,4 & Insatisfatória & Insatisfatória \\
\hline Caapiranga & 100,0 & 100,0 & 8,1 & 0,3 & $1.314,8$ & 0,5 & 194,2 & 2,7 & $4.180,4$ & 0,3 & 3,5 & 1,5 & Intermediária & Intermediária \\
\hline Canutama & 66,1 & 100,0 & 5,7 & 12,6 & 546,5 & 2,8 & 336,3 & 38,5 & 858,1 & 0,3 & 2,9 & 1,8 & Intermediária & Intermediária \\
\hline Carauari & 0,0 & 0,0 & 0,0 & 0,0 & 0,0 & 0,0 & 0,0 & 0,0 & 0,0 & 0,0 & 6,0 & 3,9 & Insatisfatória & Insatisfatória \\
\hline Fonte Boa & 59,7 & 60,3 & 5,0 & 1,3 & 3,4 & 0,2 & 0,7 & 35,8 & 3,2 & 0,3 & 2,5 & 0,0 & Satisfatória & Insatisfatória \\
\hline Guajará & 25,4 & 83,3 & $1.512,6$ & 2,5 & 2,6 & 2,9 & 56,3 & 18,3 & $1.395,0$ & 0,5 & 0,4 & 3,5 & Insatisfatória & Intermediária \\
\hline Humaitá & 21,9 & 82,5 & 43,8 & 11,6 & $1.779,4$ & 0,3 & 11,7 & 21,4 & 151,1 & 0,6 & 9,1 & 10,2 & Insatisfatória & Insatisfatória \\
\hline Ipixuna & 21,8 & 37,9 & 32,0 & 19,8 & 19,2 & 3,9 & 199,5 & 134,2 & $1.872,2$ & 1,0 & 3,8 & 3,4 & Insatisfatória & Insatisfatória \\
\hline Iranduba & 49,1 & 100,0 & 12,3 & 0,2 & 0,0 & 0,3 & 0,0 & 1,7 & 1,4 & 0,1 & 2,0 & 3,4 & Insatisfatória & Intermediária \\
\hline Itacoatiara & 69,1 & 96,6 & 4,8 & 0,3 & 2,5 & 0,1 & 0,3 & 4,7 & 1,4 & 0,1 & 3,8 & 7,6 & Intermediária & Insatisfatória \\
\hline Itamarati & 0,0 & 0,0 & 16,3 & 0,0 & 2,8 & 0,0 & 79,9 & 0,0 & 2,1 & 0,0 & 3,9 & 10,4 & Insatisfatória & Insatisfatória \\
\hline Itapiranga & 88,9 & 77,8 & 414,5 & 15,3 & 387,9 & 0,7 & 3,9 & 14,1 & 238,0 & 0,8 & 3,7 & 4,8 & Insatisfatória & Insatisfatória \\
\hline Japurá & 33,9 & 26,3 & 10,9 & 2,1 & $2.441,4$ & 132,7 & 10,7 & 61,0 & $1.732,8$ & 3,8 & 0,6 & 0,2 & Insatisfatória & Insatisfatória \\
\hline Juruá & 49,9 & 0,0 & 17,9 & 0,0 & 691,8 & 0,0 & 70,4 & 0,0 & 4,1 & 0,0 & 6,9 & 1,8 & Insatisfatória & Insatisfatória \\
\hline Jutaí & 0,0 & 11,9 & 0,0 & 3,2 & 0,0 & 4,5 & 0,0 & 240,1 & 0,0 & 5,5 & 0,8 & 3,1 & Insatisfatória & Insatisfatória \\
\hline Lábrea & 24,6 & 90,8 & 22,1 & 1,0 & 76,5 & 0,7 & 481,8 & 0,0 & $1.170,0$ & 0,2 & 2,6 & 3,9 & Insatisfatória & Intermediária \\
\hline Manacapuru & 40,2 & 79,7 & 2,6 & 0,7 & 5,8 & 0,2 & 0,1 & 2,2 & 1,4 & 0,1 & 1,9 & 7,4 & Insatisfatória & Insatisfatória \\
\hline Manaquiri & 26,3 & 46,7 & 6,1 & 7,7 & 0,0 & 0,1 & 0,0 & 74,1 & 6,6 & 0,9 & 1,5 & 5,7 & Insatisfatória & Insatisfatória \\
\hline Manaus & 40,1 & 34,1 & 3,2 & 0,0 & 3,7 & 0,0 & 16,4 & 0,1 & 2,4 & 0,0 & 6,3 & 3,7 & Insatisfatória & Insatisfatória \\
\hline Manicoré & 27,2 & 48,8 & 0,1 & 25,9 & 8,4 & 3,0 & 13,9 & 18,0 & 2,0 & 0,4 & 7,2 & 12,6 & Insatisfatória & Insatisfatória \\
\hline Maraã & 19,0 & 16,6 & $1.265,9$ & 1,5 & 58,7 & 1,7 & 186,5 & 19,6 & $1.459,3$ & 0,8 & 11,5 & 16,1 & Insatisfatória & Insatisfatória \\
\hline
\end{tabular}

(continua) 


\begin{tabular}{|c|c|c|c|c|c|c|c|c|c|c|c|c|c|c|}
\hline \multirow[t]{3}{*}{ Municípios } & \multirow{2}{*}{\multicolumn{2}{|c|}{$\begin{array}{l}\text { Cobertura } \\
\text { populacional } \\
\text { potencial } \\
\text { (\%) }\end{array}$}} & \multicolumn{8}{|c|}{ Dimensão mudança * } & \multirow{2}{*}{\multicolumn{2}{|c|}{$\begin{array}{l}\text { Dimensão impacto } \\
\text { Número de } \\
\text { internações/ } \\
1.000 \text { habitantes }\end{array}$}} & \multirow{2}{*}{\multicolumn{2}{|c|}{$\begin{array}{l}\text { Classificação da } \\
\text { implantação }\end{array}$}} \\
\hline & & & \multicolumn{2}{|c|}{$\begin{array}{l}\text { Razão da visita } \\
\text { domiciliar do } \\
\text { médico }\end{array}$} & \multicolumn{2}{|c|}{$\begin{array}{l}\text { Razão de } \\
\text { exames de } \\
\text { patologia } \\
\text { clínica }\end{array}$} & \multicolumn{2}{|c|}{$\begin{array}{c}\text { Razão de } \\
\text { encaminhamento } \\
\text { ao especialista }\end{array}$} & \multicolumn{2}{|c|}{$\begin{array}{c}\text { Razão de } \\
\text { atendimento } \\
\text { de } \\
\text { enfermagem }\end{array}$} & & & & \\
\hline & 2004 & 2008 & 2004 & 2008 & 2004 & 2008 & 2004 & 2008 & 2004 & 2008 & 2004 & 2008 & 2004 & 2008 \\
\hline Maués & 49,2 & 78,9 & 25,0 & 3,2 & 5,6 & 0,2 & 0,1 & 13,1 & 2,7 & 0,2 & 3,2 & 4,2 & Insatisfatória & Insatisfatória \\
\hline Nhamundá & 43,7 & 61,4 & 5,4 & 0,92 & $2.026,2$ & 0,3 & 20,3 & 407,4 & 8,2 & 0,6 & 0,9 & 0,9 & Insatisfatória & Intermediária \\
\hline Nova Olinda do & 26,6 & 90,3 & 6,8 & 0,5 & 98,2 & 0,7 & 0,0 & 41,8 & $2.222,5$ & 0,2 & 6,5 & 4,7 & Insatisfatória & Insatisfatória \\
\hline Norte & & & & & & & & & & & & & & \\
\hline Novo Airão & 0,0 & 100,0 & 0,0 & 5,9 & 0,0 & 1,8 & 0,0 & 12,0 & 0,0 & 1,0 & 4,8 & 18,0 & Insatisfatória & Insatisfatória \\
\hline Novo Aripuanã & 0,0 & 63,4 & 0,0 & 0,7 & 0,0 & 0,4 & 0,0 & 36,3 & 0,0 & 0,2 & 14,0 & 19,0 & Insatisfatória & Insatisfatória \\
\hline Parintins & 32,1 & 62,2 & 9,1 & 0,6 & 0,2 & 0,2 & 0,1 & 3,1 & 1,4 & 0,1 & 4,4 & 6,2 & Insatisfatória & Insatisfatória \\
\hline Pauini & 20,2 & 59,0 & 901,0 & 1,0 & 44,3 & 1,1 & 11,0 & 142,3 & 2,5 & 0,4 & 2,2 & 3,0 & Insatisfatória & Intermediária \\
\hline Presidente & 52,9 & 89,4 & 22,0 & 2,9 & 5,9 & 0,5 & 0,0 & 2,4 & 2,5 & 0,3 & 0,9 & 6,1 & Satisfatória & Insatisfatória \\
\hline Figueiredo & & & & & & & & & & & & & & \\
\hline Rio Preto da Eva & 52,0 & 100,0 & $1.682,7$ & 0,53 & $3.601,1$ & 0,5 & 16,3 & 8,0 & $2.390,9$ & 0,1 & 2,6 & 3,6 & Intermediária & Intermediária \\
\hline Santa Isabel do & 72,3 & 48,8 & $1.353,2$ & 13,2 & 7,9 & 8,3 & 715,8 & 231,3 & $2.140,0$ & 1,4 & 6,2 & 5,1 & Insatisfatória & Insatisfatória \\
\hline Rio Negro & & & & & & & & & & & & & & \\
\hline $\begin{array}{l}\text { Santo Antônio } \\
\text { do Içá }\end{array}$ & 0,0 & 0,0 & 0,0 & 0,0 & 0,0 & 0,0 & 0,0 & 0,0 & 0,0 & 0,0 & 0,0 & 10,1 & Insatisfatória & Insatisfatória \\
\hline São Gabriel da & 44,0 & 39,6 & 5,4 & 2,0 & $1.292,3$ & 0,2 & 9,0 & 5,7 & $1.525,6$ & 0,5 & 12,6 & 6,0 & Insatisfatória & Insatisfatória \\
\hline Cachoeira & & & & & & & & & & & & & & \\
\hline São Paulo de & 0,0 & 0,0 & 0,0 & 0,0 & 0,0 & 0,0 & 0,0 & 0,0 & 0,0 & 0,0 & 2,2 & 7,7 & Insatisfatória & Insatisfatória \\
\hline Olivença & & & & & & & & & & & & & & \\
\hline São Sebastião do & 100,0 & 100,0 & 3,4 & 6,6 & 91,1 & 0,4 & 0,0 & 0,0 & 314,2 & 0,6 & 8,4 & 5,9 & Insatisfatória & Insatisfatória \\
\hline Uatumã & & & & & & & & & & & & & & \\
\hline Silves & 100,0 & 74,4 & 489,1 & 13,2 & 1,1 & 10,7 & 0,0 & 131,2 & 179,2 & 0,8 & 3,4 & 4,2 & Intermediária & Intermediária \\
\hline Tabatinga & 60,3 & 61,2 & 15,9 & 2,4 & 6,8 & 0,9 & 0,3 & 10,9 & 0,9 & 0,1 & 4,2 & 1,7 & Insatisfatória & Intermediária \\
\hline Tapauá & 88,1 & 100,0 & 6,5 & 1,9 & 8,2 & 0,8 & 0,1 & 30,9 & 2,1 & 0,3 & 2,2 & 4,1 & Intermediária & Intermediária \\
\hline Tefé & 0,0 & 37,1 & 2,2 & 5,5 & 28,9 & 0,3 & 0,0 & 31,8 & 5,1 & 0,3 & 5,4 & 7,6 & Insatisfatória & Insatisfatória \\
\hline Tonantins & 0,0 & 71,0 & 4,7 & 0,3 & 53,9 & 1,7 & 75,4 & 241,4 & 14,0 & 0,2 & 5,4 & 3,1 & Insatisfatória & Intermediária \\
\hline Uarini & 0,0 & 96,7 & 0,0 & 1,0 & 0,0 & 1,2 & 0,0 & 179,1 & 0,0 & 0,3 & 0,0 & 3,2 & Insatisfatória & Intermediária \\
\hline Urucará & 34,8 & 88,4 & 14,4 & 3,3 & 5,1 & 0,3 & 41,9 & 20,8 & 11,3 & 0,2 & 6,2 & 4,6 & Insatisfatória & Intermediária \\
\hline Uricurituba & 31,0 & 100,0 & 5,3 & 2,9 & 900,2 & 1,0 & 29,4 & 54,3 & 16,0 & 1,4 & 5,3 & 9,9 & Insatisfatória & Insatisfatória \\
\hline
\end{tabular}

Fontes: Sistema de Informação da Atenção Básica e Sistema de Informações Hospitalares do Departamento de Informática do SUS.

* Conforme detalhado na Figura 1.

cobertura alta; já em 2008, houve uma mudança, principalmente na diminuição dos municípios com baixa cobertura, passando para 29 municípios, e no aumento do alto índice de cobertura, chegando a 23 municípios. Essa mudança foi estatisticamente significante.

Para a dimensão de mudança no modelo assistencial comparando-se os dois períodos, houve um aumento de 56 para 61 no número de municípios com classificação fraca no índice de cobertura. Na classificação média, houve diminuição de 6 para 1 no número de municípios.
Em toda esta dimensão, não foram encontradas diferenças estatisticamente significantes.

Para a dimensão impacto, ocorreram diferenças significantes de 2004 a 2008: houve diminuição de municípios na classificação baixa de impacto e aumento de 16 para 33 municípios para a classificação média. Entretanto, na classificação alta de impacto, não houve mudança entre os dois anos em estudo.

Apesar da diminuição do número de municípios com classificação de implantação insatisfatória e o aumento daqueles com classificação 
Classificação por indicadores.

\begin{tabular}{|c|c|c|c|c|c|}
\hline \multirow[t]{2}{*}{ Classificação } & \multicolumn{2}{|c|}{2004} & \multicolumn{2}{|c|}{2008} & \multirow{2}{*}{$\begin{array}{c}\text { Valor de } p \\
\chi^{2}\end{array}$} \\
\hline & $\mathrm{n}(\mathrm{RP})$ & $\%$ & $\mathrm{n}(\mathrm{RP})$ & $\%$ & \\
\hline \multicolumn{6}{|l|}{ Cobertura } \\
\hline Baixa & $46(3,12)$ & 74,2 & $29(-3,12)$ & 46,8 & 0,003 \\
\hline Média & $8(-0,51)$ & 12,9 & $10(0,51)$ & 16,1 & \\
\hline Alta & $8(-3,11)$ & 12,9 & $23(3,11)$ & 37,1 & \\
\hline \multicolumn{6}{|c|}{ Indicio de mudança no modelo assistencial } \\
\hline Fraca & $56(-1,95)$ & 90,3 & $61(1,95)$ & 98,4 & 0,05 * \\
\hline Média & $6(1,95)$ & 9,7 & $1(-1,95)$ & 1,6 & \\
\hline \multicolumn{6}{|l|}{ Impacto } \\
\hline Baixa & $35(3,47)$ & 56,5 & $16(-3,47)$ & 25,8 & 0,001 \\
\hline Média & $16(-3,12)$ & 25,8 & $33(3,12)$ & 53,2 & \\
\hline Alta & $11(-0,45)$ & 17,7 & $13(0,45)$ & 21,0 & \\
\hline \multicolumn{6}{|c|}{ Classificação da implantação } \\
\hline Insatisfatória & $50(1,01)$ & 80,7 & $45(-1,01)$ & 72,6 & 0,13 * \\
\hline Intermediaria & $10(-1,52)$ & 16,1 & $17(1,52)$ & 27,4 & \\
\hline Satisfatória & $2(1,43)$ & 3,2 & $0(-1,43)$ & 0,0 & \\
\hline
\end{tabular}

RP: resíduo padronizado.

Fonte: Sistema de Informação da Atenção Básica e Sistema de informações hospitalares, Departamento de Informática do SUS.

Nota: em negrito estão as categorias que apresentaram resíduo padronizado do teste qui-quadrado com valor positivo maior ou igual a 1,96, que corresponde ao nível de significância para o excesso de ocorrências.

* Teste exato de Fisher.

de implantação intermediária, o teste estatístico indica que não houve mudança significante entre um ano e outro na classificação final de implantação no Estado de Amazonas.

Os mapas de 2004 e 2008 (Figura 2) apresentam a distribuição desses municípios conforme a classificação de implantação: insatisfatória, intermediária e satisfatória. São destacados os municípios com classificação intermediária ou satisfatória. Nota-se que ocorreu aumento nos municípios com classificação intermediária de implantação, porém o mesmo não aconteceu com os municípios com classificação satisfatória. É possível, ainda, verificar que a faixa do mapa com concentração de municípios com implantação intermediária é maior ao sul do Amazonas.

\section{Discussão}

Esta análise permitiu identificar a situação de implantação do PSF no Amazonas, cuja realidade observada não aponta mudanças expressivas, prevalecendo a baixa cobertura populacional potencial. Tal fato se contrapõe ao estudo que ser- viu de base metodológica a esta análise, realizado nos anos de 2001 e 2004 no Estado de Santa Catarina 3 , onde houve mudanças significativas, com alta cobertura populacional potencial, possivelmente motivada pelo financiamento oferecido aos municípios por equipe de PSF implantada.

$\mathrm{O}$ indicador de cobertura populacional do Estado do Amazonas ainda não tem implantação total de municípios com as ações do PSF. Quanto a isso, há que se considerar que a cobertura analisada neste trabalho utiliza, no denominador, a constante de 3.450 por equipe de saúde da família. Contudo, uma abordagem mais adequada seria levar-se em conta a população de cada município e sua distribuição geográfica em âmbito urbano e rural, pois sabe-se que as distâncias e o contexto amazônico são muito peculiares. Sendo assim, não se tem conhecimento se a constante utilizada é de fato adequada a essa realidade. Como esta análise acurada não foi aqui realizada, torna-se um fator limitante do adequado julgamento da cobertura do PSF. No entanto, como parâmetro de comparação nacional e com outros estudos que utilizam esse mesmo fator, é plausível a utilização da proposta do Ministério da Saúde. 
Figura 2

Classificação de implantação do Programa Saúde da Família nos municípios do Amazonas, Brasil, em 2004 e 2008.
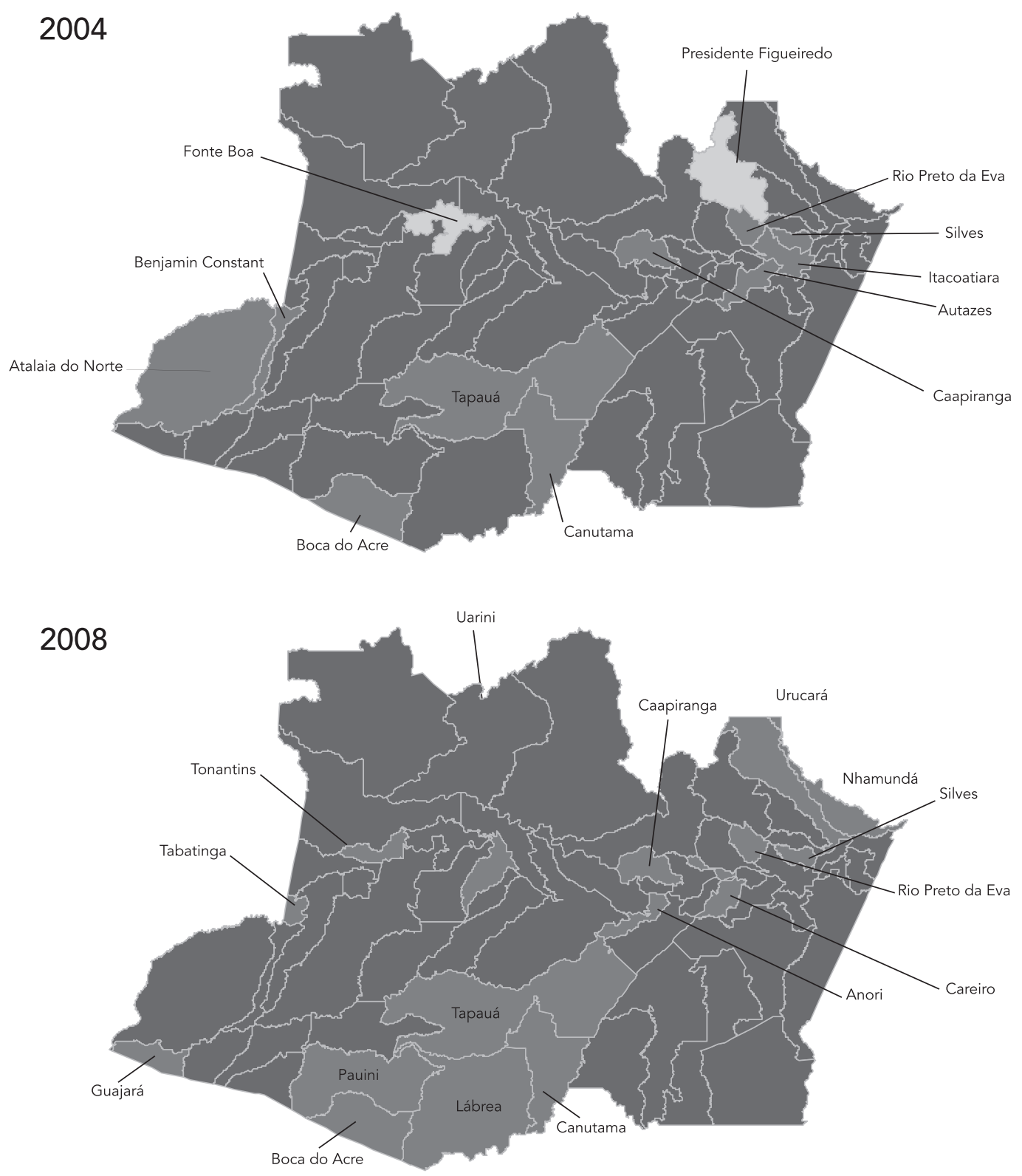
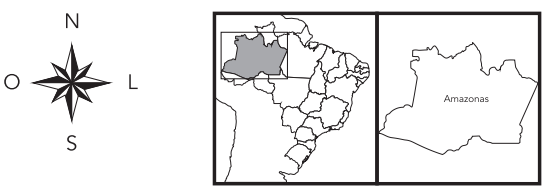

Classificação

Insatisfatória

$\square$ Intermediária

Satisfatório

800 
Tendo em conta essa limitação, a análise dos dados dos anos pesquisados mostra aumento tanto no número de municípios com implantação do PSF, como na cobertura do programa, porém ainda abaixo de $50 \%$ do total cobertura populacional. Isso é fato preocupante, uma vez que as distâncias geográficas dos pólos de serviços de saúde deixam muitas pessoas sem assistência básica, gerando outros problemas de saúde para a população desassistida por este nível de atenção em todas as faixas etárias.

O PSF surgiu com o propósito de ampliar o acesso da população aos serviços básicos de saúde, como uma possibilidade de mudança do modelo assistencial vigente. Todavia, por estar no contexto do SUS, não há como se distanciar dos seus dilemas, quais sejam: prestar assistência universal e igualitária, não obstante a realidade desigual inter e intramunicípios 6. Desse modo, torna-se um grande desafio ao PSF não somente ampliar a sua cobertura, mas que também dar conta daquilo que é proposto na atenção primária à saúde.

Um estudo comparando as regiões Sul e Nordeste 7 mostra que, no Sul, o PSF necessita de uma forte expansão para alcançar uma cobertura populacional de $60 \%$, enquanto no Nordeste é preciso consolidar o aumento crescente da cobertura. Nesse processo, a maior precarização do trabalho promovida pelo PSF, principalmente no Nordeste, representa um aspecto político da estratégia que deve ser enfrentado para não comprometer seus benefícios 8 .

O aumento no índice de municípios com cobertura do PSF não significa que a população está sendo assistida dentro dos princípios que regem o SUS 1, porém, levando em consideração a extensão territorial e as peculiaridades da região do Estado do Amazonas, esse aumento na cobertura é essencial para diminuição das dificuldades de acesso da população aos serviços de saúde.

No que se refere à dimensão de mudanças no modelo assistencial, Santa Catarina apresentou um fraco indício, que se manteve nos dois períodos estudados. Este fato indica que os municípios do Estado de Santa Catarina, não dispunham de um modelo assistencial que abrangesse de forma adequada os aspectos analisados 3 .

No Amazonas também prevaleceu a classificação fraca de indício de mudança, talvez pelo mesmo motivo, o que é preocupante, pois espera-se que, com a atuação do PSF, o foco preventivo da atenção primária seja capaz de dar conta das necessidades da população. Tal classificação pode ser indicativa de que as ações da equipe não estão em consonância com a proposta do programa, de promoção de saúde e de fortalecimento da atenção básica. É possível, ainda, que seja decorrente da maneira pela qual o profissional foi inserido no programa, das suas motivações profissionais e das dificuldades em sua formação, voltada para a saúde da família. As ações da equipe multiprofissional precisam ser pautadas, de acordo com o programa, na assistência, no planejamento de ações e estratégias capazes de prevenir os agravos à saúde, cujo foco deve ser a família e a coletividade 9 .

Na dimensão impacto, verificou-se que houve mudança em Santa Catarina, porém pouco significante estatisticamente. Quando se compara ao resultado encontrado para o Amazonas, verificase que neste estado, entre 2004 e 2006, diminuiu a quantidade de municípios na classificação baixa e aumentou aqueles na classificação média.

Isso pode demonstrar que, apesar da complexidade de implantação do PSF no Amazonas, os registros de internação por doenças sensíveis à atenção primária diminuíram. Todavia, com este estudo, não é possível sustentar esta afirmação categoricamente, pois as internações são influenciadas por uma série de questões que aqui não foram analisadas, constituindo-se em limitação para uma interpretação mais apropriada. Não se sabe se o município internou menos pacientes em virtude de sua própria capacidade hospitalar; além disso, neste indicador não estão incluídas as informações da estrutura etária da população, a qual pode influenciar sobremaneira na taxa de internação.

No tocante às doenças sensíveis à atenção primária, há necessidade de ampliação da discussão, visto que a escolha de análise dessas doenças deve levar em consideração as políticas de atenção básica e de admissão hospitalar, a prevalência das doenças e o comportamento da população na procura aos serviços de saúde. Neste estudo, entretanto, as doenças consideradas foram as mesmas analisadas por Henrique \& Calvo ${ }^{3}$. Para uma análise mais acurada, deveria haver uma validação para a realidade do Amazonas, o que não foi foco neste estudo. Ainda assim, as doenças selecionadas coincidem com as primeiras causas de internação em um estudo que buscou uma lista brasileira de tais doenças a fim de medir o desempenho do sistema de saúde 10 .

Em um trabalho sobre o papel do PSF na organização da atenção primária em sistemas municipais de saúde 11, é destacada a diferença entre as ações executadas pela equipe da Estratégia Saúde da Família no contexto da zona urbana e rural, onde distância, tamanho do território e população foram fatores limitantes para um bom desempenho do programa, mesmo em condições políticas favoráveis. Reforça-se, assim, a idéia de que o PSF não está positivamente a favor da população rural, visto que ainda não foram venci- 
das as barreiras limitantes à completa implantação do programa no estado em que é fortemente latente a questão de territorialização.

Os indicadores de ações preventivas e de promoção da saúde inerentes à atenção primária à saúde e os de morbidades específicas na população de abrangência são essenciais para a avaliação da dimensão do impacto do PSF. O adequado enfrentamento dos principais problemas do PSF poderá modificar e melhorar o seu desempenho e causar impacto positivo na saúde da população 7 .

Alguns autores 12,13 destacam que a avaliação de um programa de saúde indica que este se relaciona com a mudança no estado de saúde, atual ou futuro, do paciente, podendo ser atribuída aos cuidados à saúde recebidos previamente. Acrescentam que, para a abordagem de resultados, é essencial o desenvolvimento de indicadores do nível de saúde.

A pesquisa Avaliação de Qualidade da Atenção Pré-natal em Quatro Unidades do PSF do Município de Manaus, Amazonas mostra que o trabalho da assistência ao pré-natal das quatro unidades do Programa Médico da Família de Manaus tem resultados fracos, "pois não atinge padrões razoáveis de efetividade normatizados e recomendados pelo Ministério da Saúde e nem em níveis de adequação satisfatórios quando comparados com outros estudos realizados sobre atenção pré-natal" 14 (p. 58). Isso corrobora o achado desta investigação quanto à necessidade da efetiva mudança no modelo assistencial, o qual deve ser comprometido com a atenção básica.

Na classificação final dos municípios de Santa Catarina, foram identificadas mudanças estatisticamente significantes, mesmo com baixo impacto. Por sua vez, os municípios do Estado do Amazonas não apresentaram mudanças significantes em todos os indicadores pesquisados, com um alto grau de classificação insatisfatória.

Algo impressionante ocorreu com os municípios de Fonte Boa e Presidente Figueiredo: em 2004, eram os únicos a terem implantação satisfatória, porém esta classificação mudou para insatisfatória em 2008, mesmo o segundo sendo próximo de Manaus (cerca de $100 \mathrm{~km}$ ). O fato é que a própria capital, nos períodos estudados, apresentou grau de implantação insatisfatório, talvez por influência política no estado ou por o início da implantação do programa não ter se dado conforme o preconizado pelo Ministério da Saúde.

Os municípios ao sul do Amazonas foram mais prevalentes na classificação intermediária. Com este tipo de análise, não é possível precisar a razão desse resultado, mas talvez o fato de se situarem geograficamente em regiões que permitem o fácil acesso à capital diminua os problemas de saúde notificados nesses municípios.
Embora as análises de dados secundários por si sós tenham as limitações de não se ter o controle no registro dos dados, a subnotificação ou sobrenotificação não foram mensuradas nesta análise. Considera-se que os dados de cobertura de PSF retratem a realidade, pois têm sido frequentemente alvos de monitoramento pelo Ministério da Saúde e servem de base para os repasses orçamentários aos municípios. Os demais dados utilizados estão sujeitos a falhas conforme a sua forma de preenchimento, com fins burocráticos e não de pesquisa, dificuldades de comunicação para envio informatizado de informação ao sistema de informação, entre outras. Contudo, considera-se que esta análise permitiu uma aproximação e idéia geral da situação de implantação do PSF no Amazonas.

\section{Considerações finais}

A caracterização da implantação do PSF nos municípios do Estado do Amazonas, analisando-se os anos de 2004 e 2008, por meio de indicadores representativos de cobertura, indícios de mudança do modelo assistencial e impacto, sinalizou que a implantação do PSF classifica-se como insatisfatória em todo o estado. Isso demonstra a fragilidade do programa, que tem como princípio definido um novo modo de atender as necessidades de saúde da população.

Pautado nas diretrizes de integralidade, equidade e universalidade do SUS, o PSF propõe nova dinâmica para a estruturação dos serviços de saúde, sendo considerado uma estratégia importante na transformação do atual modelo assistencial 8,15.

A avaliação do PSF não é algo simples, pois há vários fatores que contribuem para essa problemática, como burocratização dos serviços, unidades locais improvisadas, equipe com sobrecarga de trabalho, falta de integração do PSF com outros programas municipais, dentre outros fatores ${ }^{8}$. Há muitos desafios a serem vencidos pelo programa, entre eles o de como qualificar e classificar uma família, consolidando as informações provenientes de seus membros, de modo a compreender sua organização. Ademais, ele deve reconstruir as práxis humanas, reinventando maneiras de ser no âmbito familiar e comunitário 7 .

Outros estudos poderão revelar os fatores para a classificação insatisfatória do PSF no Estado do Amazonas para que os gestores tenham parâmetros para tomada de decisão e maior efetividade e consolidação do programa em todo o estado. 


\section{Resumo}

Este artigo aborda a implantação do Programa Saúde da Família (PSF) em municípios do Estado do Amazonas, Brasil, analisando indicadores de cobertura populacional potencial, indícios de mudança do modelo assistencial e indício de impacto (2004 e 2008). A classificação de implantação foi intermediária e insatisfatória. Os resultados mostram aumento no número de municípios com PSF implantado, assim como na cobertura do programa, porém ainda abaixo de $50 \%$ da cobertura populacional. Para a dimensão de mudança no modelo assistencial, não foram observados municípios com alto índice de mudança. No indício de impacto houve diminuição no baixo impacto e aumento do alto impacto. $O$ teste estatístico indica que a classificação final não apresentou mudança estatisticamente significante entre um ano e outro, prevalecendo nos municípios do Estado do Amazonas a classificação de implantação insatisfatória. A avaliação do PSF não é algo simples, pois há vários fatores que contribuem para essa problemática. Outros estudos poderão revelar os fatores para sua classificação insatisfatória no Estado do Amazonas.

Programa Saúde da Família; Avaliação de Programas; Avaliação em Saúde; Cobertura de Serviços de Saúde

\section{Colaboradores}

H. M. Oliveira coletou e analisou os dados, fez a revisão da literatura e escreveu o manuscrito. M. J. F. Gonçalves coletou e analisou os dados e participou da discussão, redação e revisão final do artigo. R. O. M. Pires analisou os dados e participou da discussão, redação e revisão final do artigo.

\section{Referências}

1. Secretaria de Políticas Públicas, Ministério da Saúde. Guia prático do Programa Saúde da Família. Brasília: Ministério da Saúde; 2001.

2. Rosa WAG, Labate RC. Programa saúde da família: a construção de um novo modelo de assistência. Rev Latinoam Enferm 2005; 13:1027-34.

3. Henrique F, Calvo MC. Avaliação do Programa Saúde da Família nos municípios do Estado de Santa Catarina, Brasil. Cad Saúde Pública 2008; 24: 809-19.

4. Organização Mundial da Saúde. Classificação estatística internacional de doenças e problemas relacionados à saúde, 10a revisão. São Paulo: Centro Colaborador da Organização Mundial da Saúde para a Classificação de Doenças em Português; 1995.

5. Pereira JCR. Análise de dados qualitativos: estratégias metodológicas para ciências da saúde, humanas e sociais. 3a Ed. São Paulo: Edusp; 2004.

6. Pires MR, Gottems LB. Análise da gestão do cuidado no Programa de Saúde da Família: referencial teórico-metodológico. Rev Bras Enferm 2009; 62:294-9.
7. Facchini LA, Piccini RX, Tomasi E, Thumé E, Silveira DS, Siqueira FV, et al. Desempenho do PSF no Sul e no Nordeste do Brasil: avaliação institucional e epidemiológica da Atenção Básica à Saúde. Ciênc Saúde Coletiva 2006; 11:669-81.

8. Favoreto CAO, Camargo Júnior KR. Alguns desafios conceituais e técnico-operacionais para o desenvolvimento do Programa de Saúde da Família como uma proposta transformadora do modelo assistencial. Physis (Rio J.) 2002; 12:59-75.

9. Noronha MGRCS, Cardoso PS, Moraes TNP, Centa ML. Resiliência: nova perspectiva na promoção da saúde da família? Ciênc Saúde Coletiva 2009; 14:497-506

10. Alfradique ME, Bonolo PF, Dourado I, Lima-Costa MF, Macinko J, Mendonça CS, et al. Internações por condições sensíveis à atenção primária: a construção da lista brasileira como ferramenta para medir o desempenho do sistema de saúde (Projeto ICSAP - Brasil). Cad Saúde Pública 2009; 25:1337-49. 
11. Medina MG, Hartz ZM. The role of the Family Health Program in the organization of primary care in municipal health systems. Cad Saúde Pública 2009; 25:1153-67.

12. Donabedian A. La calidad de la atención médica. Definición y métodos de evaluación. México DF: La Prensa Mexicana; 1984.

13. Vuori H. A qualidade da saúde. Divulg Saúde Debate 1991; 3:17-25.
14. Ribeiro Filho B. Avaliação de qualidade da atenção pré-natal em quatro unidades do Programa de Saúde da Família do município de Manaus - AM [Dissertação de Mestrado]. Rio de Janeiro: Escola Nacional de Saúde Pública, Fundação Oswaldo Cruz; 2004.

15. Trad LAB, Bastos ACS. The socio-cultural impact of the family health program: an evaluation proposal. Cad Saúde Pública 1998; 14:429-35.

Recebido em 14/Jan/2010

Versão final reapresentada em 27/Ago/2010

Aprovado em 10/Set/2010 